\title{
Anti-HMGB1 Neutralizing Antibody Ameliorates Neutrophilic Airway Inflammation by Suppressing Dendritic Cell-Mediated Th17 Polarization
}

\author{
Fang Zhang, ${ }^{1}$ Gang Huang, ${ }^{2}$ Bo Hu, ${ }^{3}$ Li-Ping Fang, ${ }^{1}$ E-Hong Cao, ${ }^{1}$ \\ Xiao-Feng Xin, ${ }^{1}$ Yong Song, ${ }^{1}$ and Yi Shi ${ }^{1}$ \\ ${ }^{1}$ Department of Pulmonary Medicine, Jinling Hospital, Nanjing University School of Medicine, Nanjing 210002, China \\ ${ }^{2}$ Department of Medical Genetics, Third Military Medical University, Chongqing 430038, China \\ ${ }^{3}$ Department of Administration, 105 Hospital, Hefei 230031, China
}

Correspondence should be addressed to Yi Shi; shiyi56@126.com

Received 17 January 2014; Revised 3 April 2014; Accepted 3 April 2014; Published 15 May 2014

Academic Editor: Anshu Agrawal

Copyright $\odot 2014$ Fang Zhang et al. This is an open access article distributed under the Creative Commons Attribution License, which permits unrestricted use, distribution, and reproduction in any medium, provided the original work is properly cited.

\begin{abstract}
We demonstrate that high mobility group box 1 protein (HMGB1) directs Th17 skewing by regulating dendritic cell (DC) function. First, our in vitro studies reveal that recombinant HMGB1 (rHMGB1) activates myeloid DCs to produce IL-23 in vitro, and rHMGB1-activated DCs prime naïve lymphocytes to produce the Th17 cytokine IL-17A. Second, we demonstrate that anti-HMGB1 neutralizing antibody attenuates HMGB1 expression, neutrophilic inflammation, airway hyperresponsiveness, and Th17-related cytokine secretion in vivo by using a murine model of neutrophilic asthma induced by ovalbumin (OVA) plus lipopolysaccharide (LPS). Furthermore, anti-HMGB1 neutralizing antibody decreases the number of Th17 cells in lung cells and suppresses the production of IL-23 by lung CD11C ${ }^{+}$APCs. Finally, we show that intranasal adoptive transfer of rHMGB1-activated DCs was sufficient to restore lung neutrophilic inflammation and the Thl7 response in a DC-driven model of asthma, whereas the transfer of rHMGB1 plus anti-HMGB1-treated mDCs significantly reduced these inflammation phenotypes. These data suggest, for the first time, that HMGB1 drives the DC-polarized Th17-type response in allergic lung inflammation and that blocking HMGB1 may benefit the attenuation of neutrophilic airway inflammation in asthma.
\end{abstract}

\section{Introduction}

Asthma is a chronic inflammatory disease of the airways that is hypothetically considered as a T helper type 2 cell- (Th2-) mediated process [1]. However, recent studies demonstrated that T-helper type 17 cell (Th17) immune responses also play roles in pathogenesis [2]. Th17 cells are a distinct subset of $\mathrm{T}$ cells that have been found to produce interleukin-17 (IL-17) and differ in function from other T cell subsets, such as Th1, Th2, and regulatory $\mathrm{T}$ cells (Treg) [3]. For example, Th 2 cells and their cytokines primarily predominate in mild to moderate allergic asthma, whereas mixed Th2 cells with a Th17 phenotype mediate severe steroid-resistant airway inflammation [4]. Furthermore, passive transfer of Ag-specific Th17 cells induced neutrophilic inflammation and airway hyperresponsiveness [5]. Th17 cells are characterized by producing IL-17 (viz. IL-17A), which is thought to cause severe asthma and induce neutrophilic inflammation [6]. However, Th17 function in severe asthma as a driving mechanism of neutrophilic inflammation is not yet fully understood and requires further exploration.

Dendritic cells (DCs) are considered the most important APCs in the initiation of the Ag-induced immune response [7]. The interaction between DCs and T cells and the existence of polarized cytokines are crucial for determining the direction of T cell polarization, such as Th1, Th2, Treg, or Th17 $[8,9]$. The polarization of Th17 cells is mainly regulated by the key polarized cytokines IL-23 and IL-6 [10]. High mobility group box 1 (HMGB1) is a DNA-binding, nuclear protein that can act as an alarmin, which is a danger signal that alerts the innate immune system to initiate the host defense [11]. Some studies demonstrate that HMGB1 stimulates DCs to 
induce the production of Th17 polarization-related factors in vitro and promotes the Th17 response in acute allograft rejection [12], experimental autoimmune myocarditis [13], and rheumatoid arthritis [14]. In our recent studies, an increase in HMGB1 expression and Th17-mediated (or Th17involved) airway inflammation have been found in a murine model of neutrophilic asthma, and HMGB1 expression in lung tissue was positively correlated with the IL-17 level or neutrophil numbers in bronchoalveolar lavage fluid (BALF) [15]. We hypothesize that HMGB1 directs Th17 skewing by regulating DC function, and HMGB1 blocking inhibits the Th17 response and Th17-mediated neutrophilic airway inflammation in asthma.

To test this hypothesis, a mouse model of neutrophilic asthma was generated using intranasal sensitization with lipopolysaccharide (LPS) plus ovalbumin (OVA) and then challenging with OVA alone. Because it is known that neutrophilic inflammation in airways is associated with bacterial LPS in the lungs of asthmatic patients [16], this model may be useful for investigating the mechanisms of Th17-mediated neutrophilic airway inflammation. The aim of this study was to evaluate whether HMGB1 promotes the Th17 response that is mediated by DCs in vitro and if blocking of HMGB1 inhibits the Th17 response and neutrophilic airway inflammation in vivo. To demonstrate the important role of HMGB1 signaling in DC-primed allergic disease, we also investigated whether adoptive transfer of HMGB1-activated DCs was sufficient to restore neutrophilic inflammation and the Th17 response in a DC-driven model of asthma. This study will uncover the immunopathological mechanisms for Th17-mediated, neutrophilic airway inflammation that is induced by HMGB1 in neutrophilic asthma.

\section{Materials and Methods}

2.1. Mice. Female Balb/C mice were obtained from the animal center of Jinling Hospital and maintained in a pathogen-free-authorized facility, where the temperature was maintained at $20-22^{\circ} \mathrm{C}$ and the humidity was kept at $50-60 \%$. The dark/light cycles were $12 \mathrm{~h}$. All animal experiments were approved by and performed in compliance with the guidelines of the Institutional Animal Care and Use Committee.

2.2. Culture and Challenge of DCs. Mouse bone marrowderived DCs (BMDCs) were generated as previously described [17]. Briefly, murine femurs and tibiae were isolated mechanically from surrounding tissues, and BM cells were flushed with medium using a syringe with a $0.45 \mathrm{~mm}$ needle. After centrifuging the cells and lysing the RBCs using ACK, $2 \times 10^{5}$ cells were cultured in complete RPMI 1640 medium in the presence of $10 \mathrm{ng} / \mathrm{mL}$ GM-CSF and $1 \mathrm{ng} / \mathrm{mL} \mathrm{IL-4.}$ After 8 days of culture, DCs were purified using anti-CD11ccoated microbeads (Miltenyi-Biotec, Auburn, CA, USA) and treated with medium in the absence or presence of various concentrations of recombinant HMGB1 (rHMGB1) (10, 100, 500 , or $1000 \mathrm{ng} / \mathrm{mL}$ ) (R\&D Systems, Minneapolis, MN) for $2 \mathrm{~d}$, or with $500 \mathrm{ng} / \mathrm{mL}$ rHMGB1 stimulation for different times $(1,2,4$, and $6 \mathrm{~d})$. rHMGB1 reagent was endotoxin tested with Limulus amebocyte lysate (ZhanJiang A\&C Biological, China) and contained $0.003 \mathrm{EU} / \mathrm{mg}$ endotoxin content. Thus, this product is considered to be endotoxin free. Also, rHMGB1 was in the disulfide form in this study. Cytokine concentrations in the cell culture supernatants were measured using enzyme-linked immunosorbent assay (ELISA) kits (IL-23 and IL-6; R\&D Systems, Minneapolis, $\mathrm{MN})$.

2.3. Coculture of DCs with $C D 4^{+} T$ Cells. CD $4^{+} \mathrm{T}$ cells were purified from the spleens of mice using a "Mouse $\mathrm{CD} 4^{+}$ T cell enrichment kit" (Stem Cell Technologies, Vancouver, BC, Canada) following the manufacturer's instructions. The purity was greater than $98 \%$. The $\mathrm{CD}^{+}{ }^{+} \mathrm{T}$ cells were then collected after incubation with anti-CD3 $(2 \mu \mathrm{g} / \mathrm{mL})$ and soluble anti-CD28 $(1 \mu \mathrm{g} / \mathrm{mL})$ in complete RPMI 1640 for 3 days and plated at $1 \times 10^{5}$ cells/well alone or cocultured with $2.5 \times 10^{4}$ autologous DCs or rHMGB1-stimulated DCs. In Transwell assays, the cells were separated by a membrane ( $6.5 \mathrm{~mm}$ diameter, $0.4 \mu \mathrm{m}$ pore size) in 24 -well plates (Costar, Cambridge, MA). The lower compartment of the wells contained rHMGB1-stimulated DCs. The upper compartments contained anti-CD3 and anti-CD28 activated $\mathrm{CD}^{+}{ }^{+} \mathrm{T}$ cells. When indicated, neutralizing Abs for IL-23p19 or control IgG (both from R\&D Systems) was added into the coculture medium at a concentration of $0.8 \mu \mathrm{g} / \mathrm{mL}$. After $5 \mathrm{~d}$ of incubation, the culture supernatants were analyzed for IL17A by ELISA, and the expression of intracellular IL-17 by $\mathrm{CD} 4^{+} \mathrm{T}$ cells was analyzed using a FACSCalibur.

2.4. Protocols for Mouse Model and Pharmacologic Intervention. A murine model of neutrophilic asthma that was characterized by Th17 cell responses was generated as described previously [18]. In brief, mice were intranasally sensitized with $75 \mu \mathrm{g}$ LPS-depleted OVA (grade V; Sigma-Aldrich, St. Louis, MO, USA) plus $10 \mu \mathrm{g}$ LPS (E. coli serotype 026 : B6; Sigma-Aldrich) on days $0,1,2$, and 7 and then challenged with $50 \mu \mathrm{g}$ OVA alone on days $14,15,21$, and 22 . One day after the final challenge, the mice were euthanized for further analyses.

In this study, the mice were divided randomly into four groups ( $n=6$ mice) as follows: (i) mice sensitized with phosphate-buffered saline (PBS) and challenged with OVA (control group); (ii) mice sensitized with OVA plus LPS and challenged with OVA (asthma group); (iii) mice treated with control IgG (R\&D Systems) for half an hour before sensitization to OVA plus LPS and the same challenge with OVA later (control IgG group); (iv) mice treated with antiHMGB1 IgG (R\&D Systems) half an hour before sensitization to OVA plus LPS and the same challenge with OVA later (anti-HMGB1 group). Anti-HMGB1 IgG or control IgG was administered intranasally $(200 \mu \mathrm{g} / \mathrm{kg})$ on days $14,15,21$, and 22 a half an hour before OVA challenge (Figure 1(a)). The dose of anti-HMGB1 IgG was predetermined using staining analysis of airway inflammation in mice receiving $100-400 \mu \mathrm{g} / \mathrm{kg}$ of anti-HMGB1 IgG.

2.5. Bronchoalveolar Lavage Fluid (BALF). One day after the challenges, the mice were anesthetized, and their BALF was 


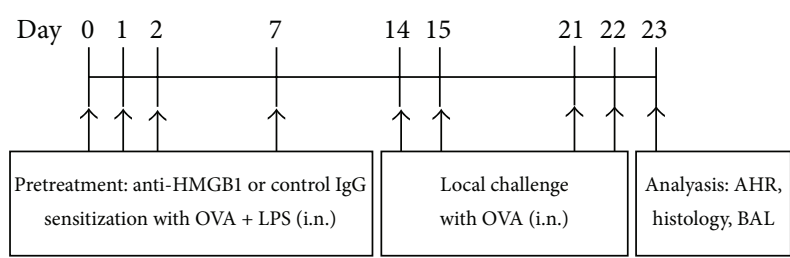

(a)

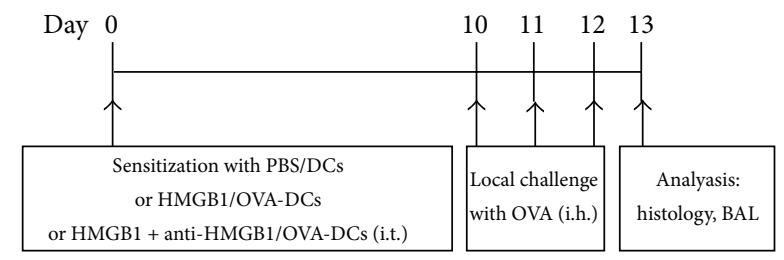

(b)

FIGURE 1: (a) OVA and LPS-induced murine model of neutrophilic asthma. Mice were intranasally sensitized with OVA plus LPS on days $0,1,2$, and 7 and then challenged with OVA on days 14, 15,21 , and 22. Anti-HMGB1 IgG or control IgG was intranasally administered a half an hour before each OVA challenge. One day after the final challenge, the mice were euthanized for further analyses. (b) A murine model of asthma by the transfer of DCs. Mice were intratracheally sensitized with PBS/DCs, HMGB1/OVA-DCs, or HMGB1 + anti-HMGB1/OVA-DCs on day 0 and then challenged with OVA on days 10,11 , and 12 . One day after the final challenge, the mice were euthanized for further analyses.

collected as described previously [19]. The cells in the BALF samples were centrifuged and loaded onto slides. Differential cell counts were ascertained in duplicate on coded slides using 200 cells from each sample. The levels of IL-23, IL-17A, and IL-4 and interferon-gamma (IFN- $\gamma$ ) in the BALF were determined using ELISA according to the manufacturer's instructions (eBioscience).

2.6. Histological Examination. After the mice were euthanized, their lungs were fixed in $4 \%$ buffered formalin, and lung tissue sections were stained with hematoxylin and eosin (H\&E). The sections were observed using a microscope at $\times 200$ magnification.

2.7. Measurement of HMGB-1 Expression. Five-micrometerthick serial sections from the paraffin-embedded lung tissue were stained to detect the expression of HMGB-1 in the lungs as described previously [20]. Briefly, the paraffinembedded sections were stained with primary antibodies to HMGB-1 (Santa Cruz Biotechnology Inc.) that was diluted at 1:50 and biotinylated secondary antibody that was diluted at 1:500. The bound peroxidase was visualized using the DAB method. We counted 10 randomly chosen fields per section using a microscope at $\times 200$ magnification, and the intensity of HMGB-1 protein staining was determined as the average optical density using IPP software (Image-Pro Plus 6.0, Media, Cybernetics). A nonstained region was selected and set as the background.
2.8. Airway Hyperreactivity (AHR). AHR was analyzed $24 \mathrm{~h}$ after the last OVA aerosol challenge. To analyze the AHR, methacholine-induced airflow obstructions from conscious mice that were placed in a whole body plethysmograph (model PLY 3211, Buxco Electronics, Troy, NY, USA) were performed, and the dimensionless parameter known as enhanced pause (Penh) was measured as previously described [19]. Briefly, the mice were placed in the plethysmograph chamber and exposed to aerosolized, normal saline solution (baseline readings) and then to cumulative concentrations of $\beta$-methacholine that ranged from 3 to $24 \mathrm{mg} / \mathrm{mL}$. The aerosol was generated using an ultrasonic nebulizer and drawn through the chamber for 2 minutes. The inlet was then closed, and Penh readings were taken for 3 minutes and averaged. The values were then reported as the Penh index.

2.9. Flow Cytometry Analysis. To detect IL- $17^{+} \mathrm{CD} 4^{+} \mathrm{T}$ cells in the DC-T cell coculture system, the cells were incubated with $10 \mu \mathrm{g} / \mathrm{mL}$ brefeldin A (eBioscience) for $2 \mathrm{~h}$ and stained with FITC-anti-CD4 mAb (eBioscience) for $30 \mathrm{~min}$ at $4^{\circ} \mathrm{C}$. After incubation with the fixation/permeabilization solution (eBioscience), the cells were stained with PE-anti-IL-17 mAb (eBioscience) for $30 \mathrm{~min}$ and analyzed using a FACSCalibur flow cytometer (BD Biosciences) and the CellQuest software.

To detect $\mathrm{IL}-17^{+} \mathrm{CD} 4^{+} \mathrm{T}$ cells in lung tissue, lung cells were obtained according to previously reported methods [21]. Briefly, the lungs were minced into small pieces and incubated in a solution containing $2 \mathrm{mg} / \mathrm{mL}$ collagenase XI and $100 \mu \mathrm{g} / \mathrm{mL}$ DNase I (both from Roche Applied Science, Indianapolis, USA) in RPMI 1640 at $37^{\circ} \mathrm{C}$ for $60 \mathrm{~min}$. EDTA ( $2 \mathrm{mM}, \mathrm{pH} 7.2$ ) was added during the last $5 \mathrm{~min}$ of incubation. Digested cells were then filtered through $70-\mu \mathrm{m}$ cell strainers, and erythrocytes were lysed using ACK lysing buffer $(150 \mathrm{mM}$ $\mathrm{NH}_{4} \mathrm{Cl}, 10 \mathrm{mM} \mathrm{KHCO}_{3}$, and $0.1 \mathrm{mM}$ EDTA). Lung cells $\left(4 \times 10^{6} / \mathrm{mL}\right)$ were incubated with brefeldin A $(10 \mu \mathrm{g} / \mathrm{mL})$ for $2 \mathrm{~h}$ and stained with surface-specific Abs (anti-CD3APC and anti-CD4-FITC; eBioscience) for $30 \mathrm{~min}$ at $4^{\circ} \mathrm{C}$. After incubation with the fixation/permeabilization solution (eBioscience), the cells were stained with PE-anti-IL-17 mAb (eBioscience) for $30 \mathrm{~min}$ and analyzed using a FACSCalibur flow cytometer.

To detect IL-23 ${ }^{+}$CD11c $^{+}$APCs in lung tissue, lowdensity lung cells were prepared as described previously [22]. Briefly, digested lung cells $\left(10 \times 10^{6} / \mathrm{mL}\right)$ were resuspended in high-density Percoll $(\rho=1.075 \mathrm{~g} / \mathrm{mL})$, overlaid with an equal volume of lower density Percoll $(\rho=1.030 \mathrm{~g} / \mathrm{mL})$, and centrifuged at $400 \times \mathrm{g}$ for $20 \mathrm{~min}$. Low-density lung cells, which were enriched for mononuclear cells, were recovered from the 1.075/1.030 Percoll interface and washed with Hanks' balanced salt solution (HBSS). Low-density lung cells were then stained with DC-specific Ab (FITC-anti-CD11C mAb, eBioscience) for $30 \mathrm{~min}$ at $4^{\circ} \mathrm{C}$. After incubation with the fixation/permeabilization solution (eBioscience), the cells were stained with PE-anti-IL-23 mAb (eBioscience) for $30 \mathrm{~min}$ and analyzed using a FACSCalibur flow cytometer. 
2.10. Adoptive Transfer of DCs. The establishment of a murine model of asthma through the transfer of bone marrowderived DCs (BMDCs) has been described [17]. Briefly, BMDCs (cultured for 8 days) were purified and pulsed with OVA overnight (OVA-DCs). OVA-DCs were then injected into anesthetized, naïve mice via the intratracheal route. Ten days after intra-tracheal immunization, the mice were challenged with OVA ( $1 \%$ w/v in PBS; grade V; Sigma-Aldrich) aerosol during a daily, 30-min challenge on 3 consecutive days. In this experiments, the mice were divided randomly into the three groups ( $n=6$ per group) as follows: (i) mice received an i.t. injection of $2 \times 10^{6} \mathrm{PBS}$-treated and nonpulsed DCs (PBS/DCs); (ii) mice received an i.t. injection of $2 \times 10^{6}$ rHMGB1-treated OVA-DCs (rHMGB1/OVA-DCs); or (iii) mice received an i.t. injection of $2 \times 10^{6}$ rHMGB1 plus antiHMGB1-treated OVA-DCs (rHMGB1 + anti-HMGB1/OVADCs). The OVA-DCs were treated with rHMGB1, antiHMGB1, or control IgG at concentrations of $500 \mathrm{ng} / \mathrm{mL}$ for $2 \mathrm{~d}$. All mice were euthanized $24 \mathrm{~h}$ after the final challenge for further analyses (Figure 1(b)).

2.11. Statistical Analysis. Data were expressed as the means \pm SD. Differences between groups were analyzed using SPSS for windows (version 16.0) and unpaired, two-tailed, parametric Student's $t$-test, or ANOVA tests. $P$ values $<0.05$ were considered statistically significant.

\section{Results}

3.1. rHMGB1-Stimulated BMDCs Induce the Th17 Response via IL-23 In Vitro. Because BMDCs express receptors for HMGB1, namely, RAGE (the receptor for advanced glycation end products), we tested whether HMGB1 could induce the production of Th17 polarization-related factors in these cells. We initially exposed BMDCs to various concentrations of rHMGB1 and measured their capacity to express IL-23 and IL-6 in culture supernatants. As shown in Figure 1(a), rHMGB1 increased the IL-23 levels in a 2-d culture, with significant effects at rHMGB1 concentrations of $500 \mathrm{ng} / \mathrm{mL}$ and $1000 \mathrm{ng} / \mathrm{mL}$ (Figure 2(a)). An increased tendency for IL6 expression was observed, but it did not reach statistical significance (data not shown). Additionally, IL-23 in culture supernatants maintained a high level at treatment with a concentration of $500 \mathrm{ng} / \mathrm{mL}$ rHMGB1 for $6 \mathrm{~d}$ (Figure 2(a)). The results indicated that rHMGB1-stimulated DCs have the potential to secrete Th17 polarization-related factors, such as IL-23, in vitro.

Because antigen-presenting cells such as DCs can modulate $\mathrm{T}$ helper cell polarization, we investigated whether rHMGB1 stimulation of BMDCs could induce the development of a Th17 phenotype in cocultured, autologous $\mathrm{CD} 4^{+}$ $\mathrm{T}$ cells. Following their 8-day culture, BMDCs were treated with $500 \mathrm{ng} / \mathrm{mL}$ rHMGB1 and cocultured with activated, autologous $\mathrm{T}$ cells for an additional 5-day period at a ratio of $1: 4$. The culture supernatants and $\mathrm{T}$ cells were then analyzed for IL-17 expression using ELISA and flow cytometry, respectively. The results showed that rHMGB1-stimulated BMDCs significantly enhanced IL-17A secretion in the culture supernatants (Figure 2(b)) and increased the percentage of IL$17^{+} \mathrm{CD}^{+} \mathrm{T}$ cells (Figure 2(c)). In contrast, activated $\mathrm{CD} 4^{+}$ $\mathrm{T}$ cells failed to upregulate their expression of IL-17 after coculturing with BMDCs. Interestingly, addition of the IL23 antibody to the coculture significantly lowered the IL-17A expression level (Figure 2(b)) and the percentage of $1 L-17^{+}$ $\mathrm{CD} 4^{+} \mathrm{T}$ cells (Figure 2(c)) that were induced by the rHMGB1stimulated BMDCs, suggesting that the IL-17 expression level was dependent upon rHMGB1 and potentially regulated by the endogenous production of IL-23 by BMDCs. In Transwell assays, the rHMGB1-stimulated BMDCs were cultured in the lower chamber, and anti-CD3 and anti-CD28 activated $\mathrm{CD}^{+}{ }^{+} \mathrm{T}$ cells were placed in the upper chamber. The results showed that the IL-17A expression level (Figure 2(b)) and the percentage of $\mathrm{IL}-17^{+} \mathrm{CD} 4^{+} \mathrm{T}$ cells (Figure 2(c)) were still mildly elevated, and significantly lowered after the addition of the IL-23 antibody to the coculture medium, suggesting that direct contact between the rHMGB1-stimulated BMDCs and preactivated $\mathrm{T}$ cells is not absolutely necessary, while the endogenous production of IL-23 is required.

\subsection{Anti-HMGB1 IgG Inhibits HMGB1 Expression In Vivo.} Because HMGB1, which is a DNA-binding factor that acts as a late inflammatory mediator, plays a major role in the maintenance of airway inflammation [23], we supposed that anti-HMGB1 IgG could decrease airway inflammation via blocking HMGB1 signaling. It was found that asthma group mice developed a significant increase in HMGB1 expression compared with control group mice $(P<0.01$, Figure 3$)$. To our surprise, the administration of anti-HMGB1 IgG resulted in a significant inhibition of HMGB1 expression compared with IgG-treated mice $(P<0.05$, Figure 3$)$, suggesting that anti-HMGB1 IgG could be an effective agent for inhibiting HMGB1 expression.

3.3. Anti-HMGB-1 IgG Suppresses Neutrophilic Airway Inflammation. To ascertain if anti-HMGB1 IgG could prevent neutrophilic airway inflammation, HMGB1 Abs were administered intranasally $(200 \mu \mathrm{g} / \mathrm{kg})$ into mice before OVA sensitization. As expected, asthma group mice exhibited significantly increased counts of total cells, neutrophils, and macrophages in their BALF compared with that of the control group mice $(P<0.05$ or 0.01 , Figure $4(a))$. However, administration of anti-HMGB1 IgG before sensitization resulted in a decrease in the total number of BALF cells, neutrophils and macrophages compared with IgG-treated mice $(P<0.05$, Figure 4(a)).

We also compared changes in lung pathology in the different groups of mice. As shown in Figure 4(b), asthma group mice showed prominent neutrophil infiltration of the peribronchiolar and perivascular connective tissues, together with the hypersecretion of mucus by epithelial cells. In contrast, the administration of anti-HMGB1 IgG markedly inhibited neutrophil infiltration and mucus hypersecretion into the airways. All of these results indicated that local blockage of HMGB1 signaling by anti-HMGB1 IgG before sensitization significantly reduced neutrophil infiltration. 

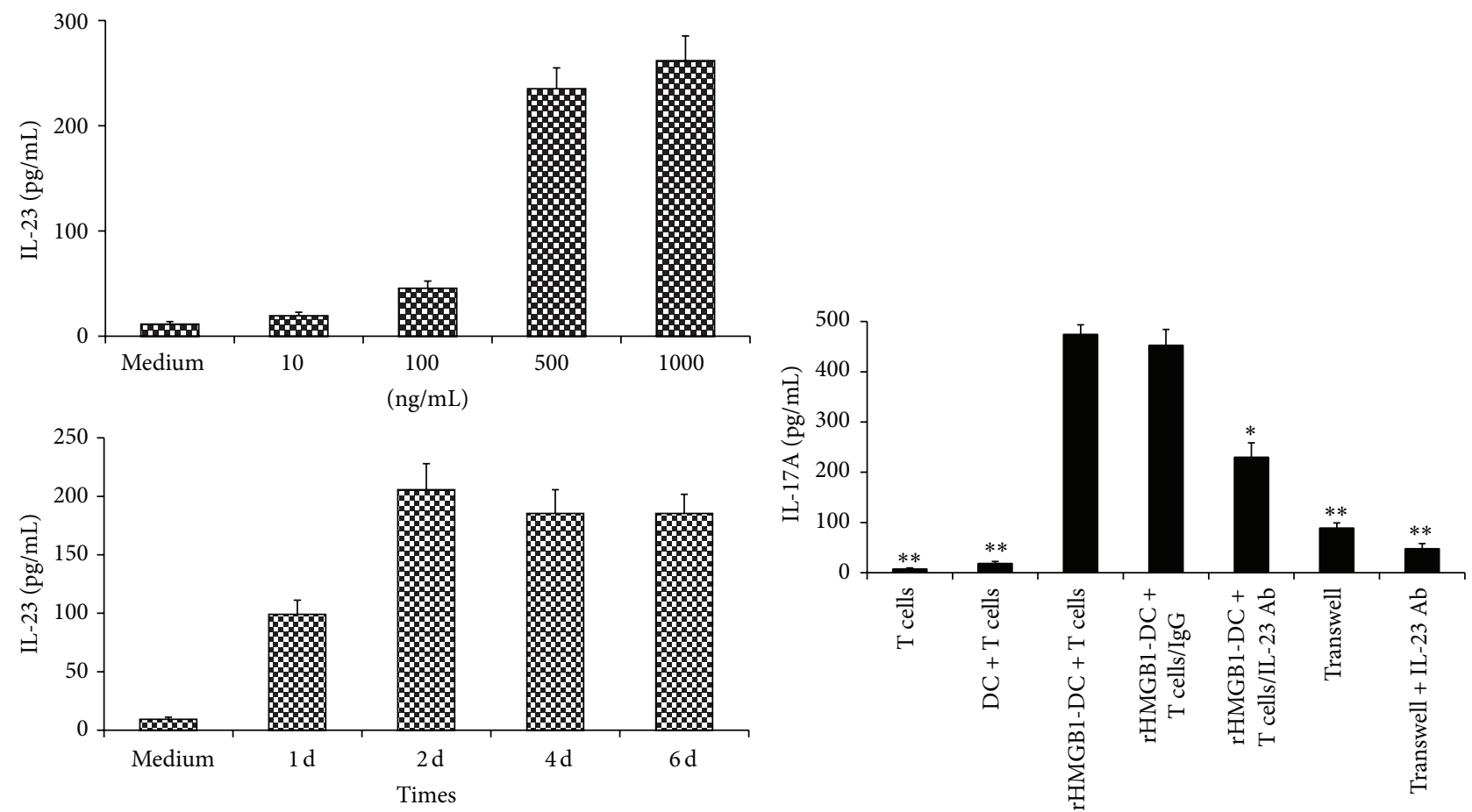

(a)

(b)
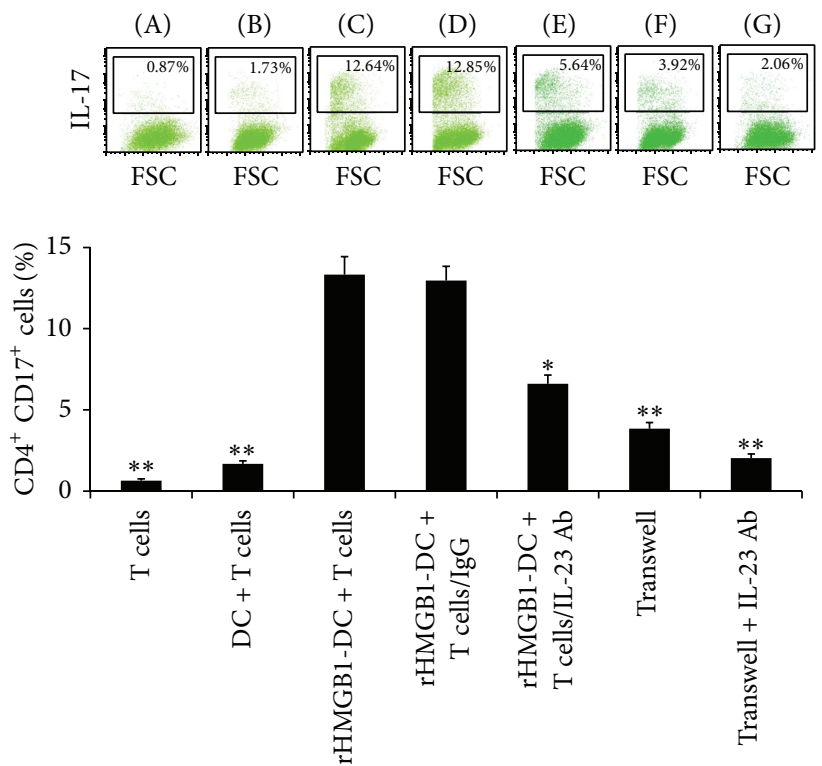

(A) (B)

(B)

(C)

(D)

(F)

(G)

(c)

Figure 2: HMGB1-stimulated BMDCs induce IL-17A production and Th17 differentiation via IL-23 in vitro. (a) HMGB1-induced IL-23 expression in BMDCs. BMDCs were stimulated with graded concentrations of HMGB1 or culture medium for $2 \mathrm{~d}$ or stimulated with $500 \mathrm{ng} / \mathrm{mL}$ HMGB1 for the indicated times. The IL-23 levels in the cell culture supernatants were measured using ELISA. The values represent the means \pm standard deviations $(n=5) .{ }^{* *} P<0.01$ compared with the medium control. (b and c) HMGB1-stimulated BMDCs modulate Th17 polarization. Activated, autologous $\mathrm{CD}^{+} \mathrm{T}$ cells $\left(1 \times 10^{5}\right)$ were cultured alone or cocultured with BMDCs $\left(2.5 \times 10^{4}\right)$ that were treated with or without $500 \mathrm{ng} / \mathrm{mL}$ HMGB1 in the presence or absence of IL-23p19 Ab or control IgG for $5 \mathrm{~d}$, the culture supernatants were analyzed for IL-17A using ELISA (b), and the expression of intracellular IL-17 by CD4 ${ }^{+} \mathrm{T}$ cells was analyzed using FACS (c). The values represent the means \pm standard deviations $(n=5) .{ }^{* *} P<0.01$ compared with the HMGB1-DC group. 


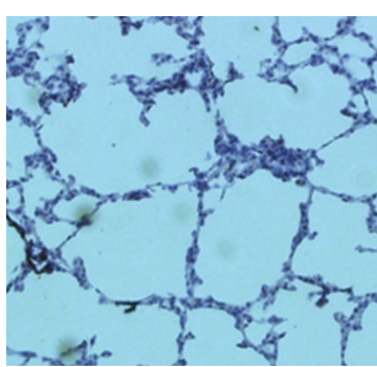

(A)

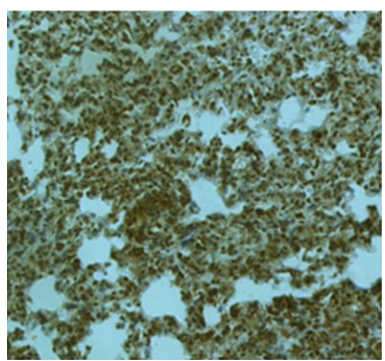

(C)

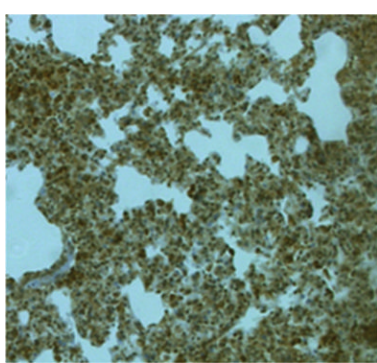

(B)

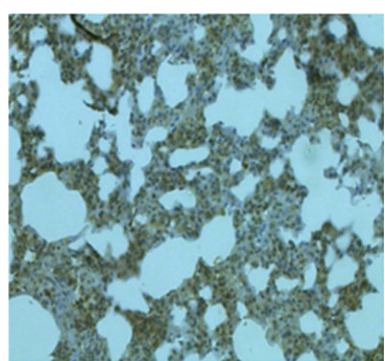

(D)

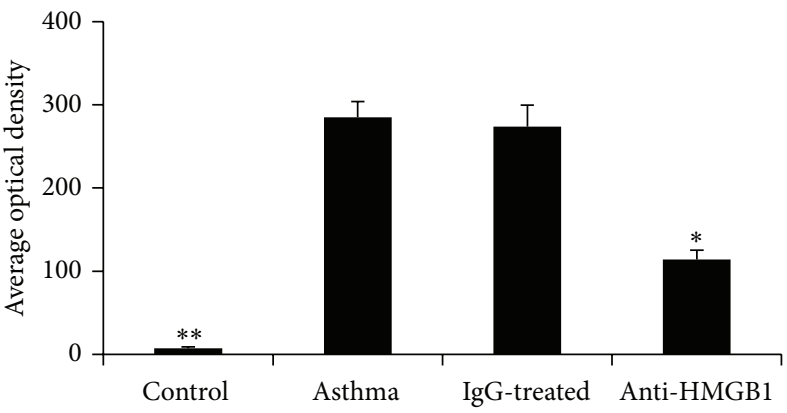

(A)

(B)

(C)

(D)

(a)

(b)

FIGURE 3: Intranasal administration of anti-HMGB1 IgG inhibited HMGB1 expression in the lung. (a) Representative images of immunohistochemical staining for HMGB1 in lung tissue from mice in the control (A), asthma (B), control IgG-treated (C), and antiHMGB1-treated groups (D). (b) The average optical density in the histogram represents the HMGB1 expression. The values represent the means \pm standard deviations $(n=5) .{ }^{*} P<0.05$ and ${ }^{* *} P<0.01$ compared with the asthma group.

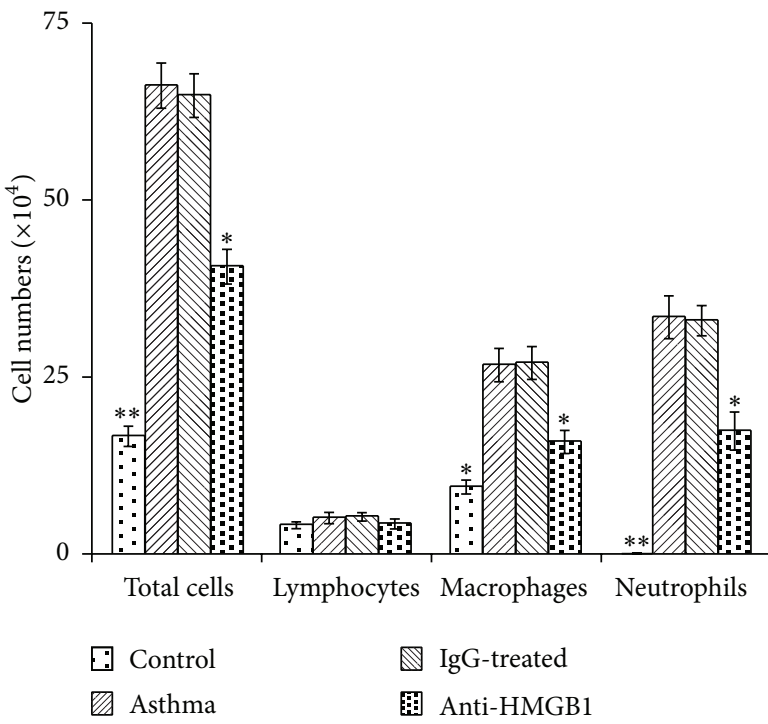

(a)

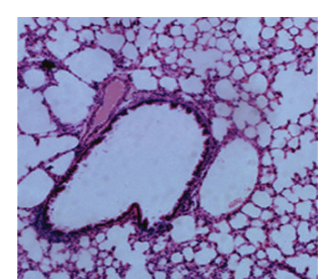

(A)

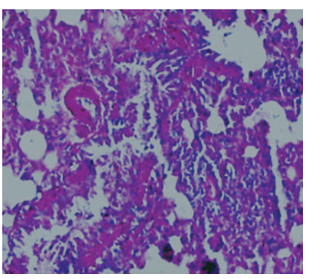

(C)

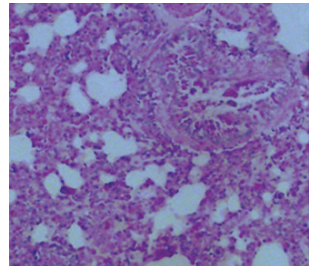

(B)

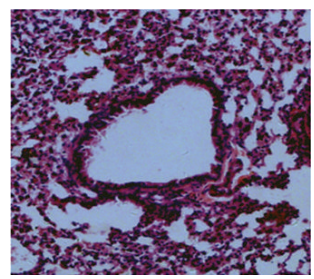

(D)

(b)

FIGURE 4: Intranasal administration of anti-HMGB1 IgG suppressed neutrophilic airway inflammation. (a) Administration of anti-HMGB1 suppressed the infiltration of neutrophils and macrophages into BALF. BALF was collected 24 hours after the last OVA challenge. The number of total and differential cells counts in the BALF of mice in the control, asthma, control IgG-treated, and anti-HMGB1-treated groups were analyzed by counting. The values represent the means \pm standard deviations $(n=5) .{ }^{*} P<0.05$ and ${ }^{* *} P<0.01$ compared with the asthma group. (b) Administration of anti-HMGB1 reduced the neutrophilic inflammation in lung tissue. Histological findings of lung tissues (original magnification, $\times 200)$ in mice from the control (A), asthma (B), control IgG-treated (C), and anti-HMGBl-treated groups (D) were analyzed using H\&E staining. 


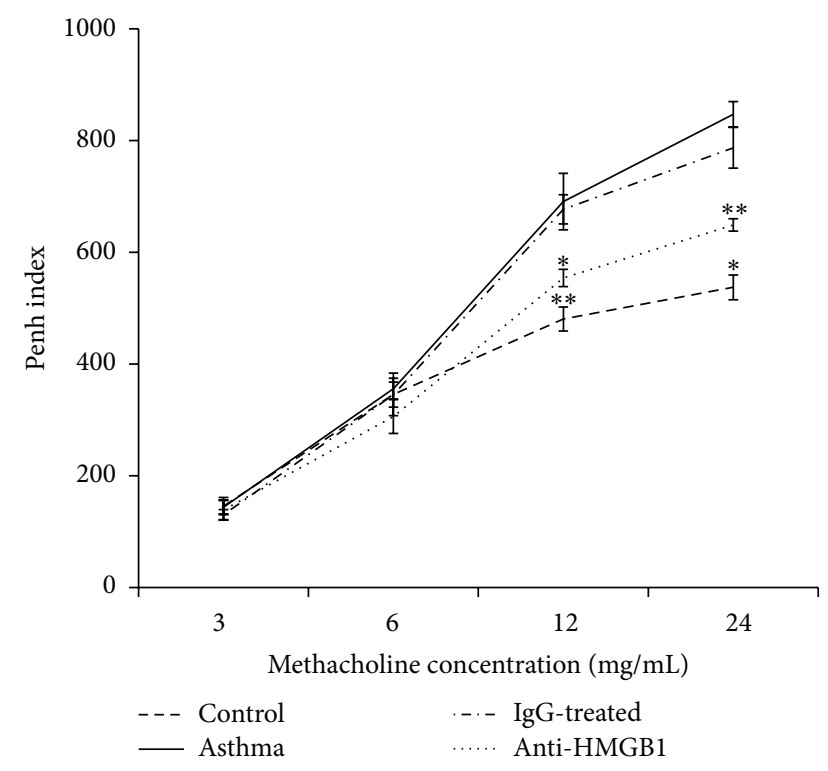

FIGURE 5: Intranasal administration of anti-HMGB1 reduced AHR to methacholine stimuli. The Penh values represent the OVAinduced AHR to methacholine and were measured in mice from the control, asthma, control IgG-treated, and anti-HMGB1-treated groups using a body plethysmograph. The values represent the means \pm standard deviations $(n=5) .{ }^{*} P<0.05$ and ${ }^{* *} P<0.01$ compared with the asthma group.

3.4. Anti-HMGB1 IgG Inhibits the Development of AHR. Next, we analyzed if administration of anti-HMGB1 IgG was important for changes in lung function. The results showed that asthma group mice developed significant AHR compared with the control group mice $(P<0.01)$ after the methacholine concentration reached $6 \mathrm{mg} / \mathrm{mL}$ (Figure 5). However, the Penh was significantly reduced in anti-HMGB1 IgG-treated mice compared with asthma group animals $(P<$ 0.05 , Figure 5$)$. These results indicated that administration of anti-HMGB1 IgG could significantly inhibit the development of AHR in the murine model of neutrophilic asthma.

3.5. Anti-HMGB1 IgG Attenuates the Th17 Response. Furthermore, we analyzed if administration of anti-HMGB1 IgG resulted in attenuation of the Th17 response. As shown in Figure 6(a), local blockage of HMGB1 led to marked reduced levels of IL-23 and IL-17A (Th17-associated cytokines) in the BALF $(P<0.05$ versus Asthma group or IgG group). In contrast, anti-HMGB1 IgG treatment decreased the level of IFN- $\gamma$ (Th1-associated cytokine) in the BALF, but this did not reach statistical significance $(P=0.064$ versus Asthma group). The level of IL-4 (Th2-associated cytokine) was not affected by anti-HMGB1 IgG treatment.

Because the level of IL-17A in BALF was increased in the asthma group mice and anti-HMGB1 IgG treatment suppressed it, we examined the possibility that this suppression was mediated through the decreased production of Th17 cells in the lung. The results indicated that the number of IL$17^{+} \mathrm{CD} 4^{+} \mathrm{T}$ cells (Th17) in the lung tissue was significantly increased in asthma group mice compared with control group

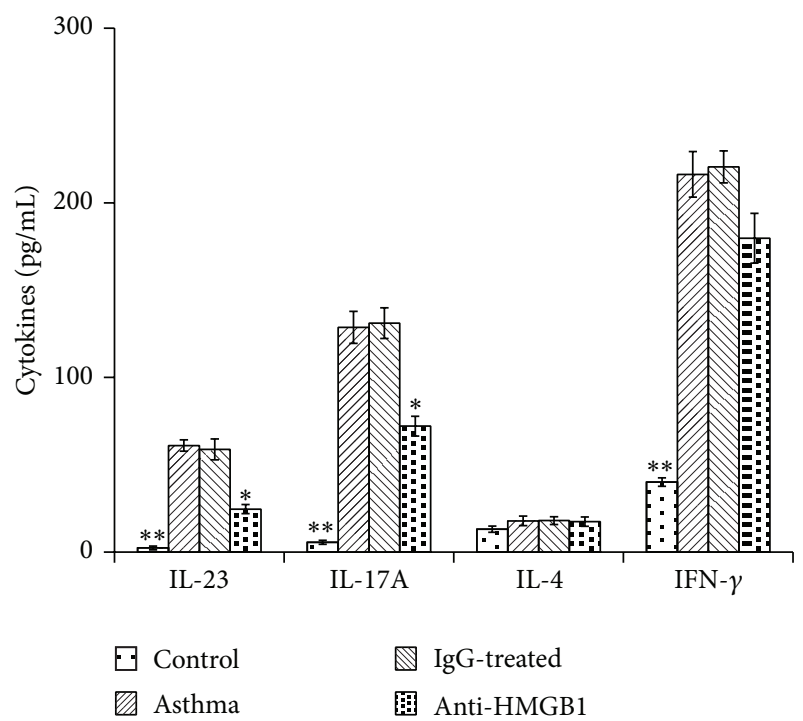

(a)

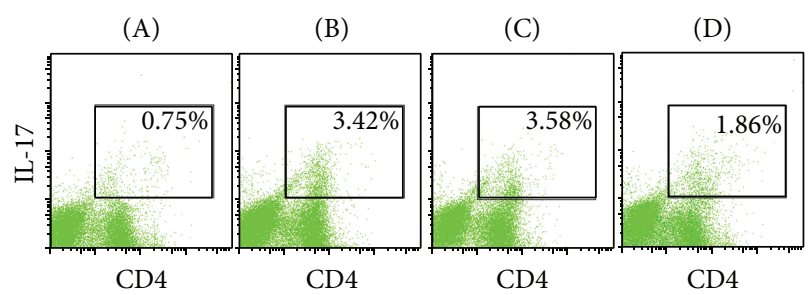

(b)

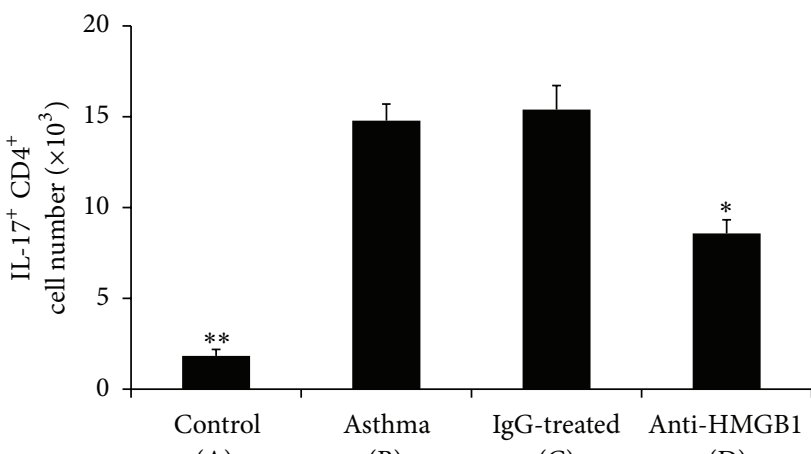

(A)

(B)

(C)

(D)

(c)

FIGURE 6: Intranasal administration of anti-HMGB1 decreased the Th17 response in the lung. (a) Administration of anti-HMGB1 decreased Th17 cytokine secretion after OVA challenge. BALFs were collected 24 hours after the last OVA challenge. The production of IL-23, IL-17A, IL-4, and IFN- $\gamma$ in the BALF of mice in the control, asthma, control IgG-treated, and anti-HMGB1-treated groups was assessed using ELISA. The values represent the means \pm standard deviations $(n=5) .{ }^{*} P<0.05$ and ${ }^{* *} P<0.01$ compared with the asthma group. (b) Administration of anti-HMGB1 downregulated the number of Th17 cells in the lung. Representative results of the percentage of Th17 cells in $\mathrm{CD}^{+}$gated lung cells from mice in the control (A), asthma (B), control IgG-treated (C), and anti-HMGB1treated groups (D) were analyzed using FACS. (c) Histogram showing the number of Th17 cells in mice from the different groups. The values represent the means \pm standard deviations $(n=5)$. ${ }^{*} P<0.05$ and ${ }^{* *} P<0.01$ compared with the asthma group. 
mice $(P<0.01$, Figure $6(\mathrm{~b}))$. As expected, the administration of anti-HMGB1 IgG significantly downregulated the number of Th17 cells in lung tissue $(P<0.05$ versus the Asthma group or IgG group, Figure $6(\mathrm{~b})$ ). These results suggested that antiHMGB1 IgG could suppress the development of Th17 cells in vivo.

3.6. Anti-HMGB1 IgG Suppresses IL-23 Production by Lung $\mathrm{CD}_{11 c^{+}}$APCs. To elucidate the mechanisms of the suppressive Th17 response, we examined the effect of anti-HMGB1 IgG on lung APC functions. Consistent with the marked reduced level of IL-23 in the BALF, the administration of antiHMGB1 IgG also significantly suppressed the production of IL-23, which is an important cytokine that is used to differentiate and promote the Th17 response [24], by lung CDIlc $^{+}$APCs $(P<0.05$ versus Asthma group or IgG group, Figure 7). These results demonstrate that anti-HMGB1 IgG could suppress the production of IL-23 by lung CD11c ${ }^{+}$ APCs, thus inhibiting the development of the Th17-mediated immune response in the lung.

3.7. Th17 Priming Induced by i.t. Injection of HMGB1Stimulated BMDCs. To clarify the impact of HMGB1 on the potential of DCs to promote Th17 skewing in vivo, we adoptively transferred rHMGB1 or rHMGB1 plus antiHMGB1 IgG-treated OVA-DCs into the airways of mice. As expected, rHMGB1/OVA-DC group mice showed a marked neutrophilic inflammation that was characterized by infiltration of neutrophils into the BALF $(P<0.01$ versus PBS/DCs group, Figure $8(\mathrm{a}))$ and Th17/Th1 priming, as shown by the increase in IL-23, IL-17A, and IFN- $\gamma$ levels in the BALF $(P<0.01$ versus PBS/DCs group, Figure 8(b)). However, rHMGB1 plus anti-HMGB1 IgG pretreatment before transferring the OVA-DCs significantly decreased the neutrophils in the BALF $(P<0.05$ versus rHMGB1/OVADCs group, Figure 8(a)) and the Th17 response, as shown by the decrease in the IL-23 and IL-17A levels $(P<0.05$ versus rHMGB1/OVA-DCs group, Figure $8(\mathrm{~b})$ ). The above data confirmed that $\mathrm{rHMGB} 1$ pretreatment promoted the capability of OVA-DCs to induce neutrophilic inflammation and Thl7 skewing in asthmatic mice.

\section{Discussion}

High mobility group box 1 (HMGB1) is a DNA binding, nuclear and cytosolic protein; in the extracellular environment, HMGB1 is an important mediator of inflammation that regulates tissue response to infection and injury [25]. In particular, HMGB1 controls the function of migratory cells from various origins $[26,27]$. Potential sources of environmental HMGB1 are dying cells or bone marrow-derived APCs [28, 29], which express HMGB1 in the nucleus and secrete it in response to inflammatory stimuli. Manfredi reported that the receptor for advanced glycation end products (RAGE), which is a multiligand member of the Ig superfamily of cellsurface molecules that acts as a receptor for HMGB1, plays a nonredundant role in DC function [30].
Our recent studies showed that HMGB1 expression and the IL-17 level in lungs increased in a murine model of neutrophilic asthma, suggesting a possible association of the inflammatory lipid mediator HMGB1 with Th17 responses. In the present study, we provide evidence that HMGB1 could effectively modulate Th17 responses. First, we found that rHMGB1 induced increased expression of IL-23 in BMDCs, which is reported to be crucial for Th17 development. Second, we observed that TCR-activated T cells in coculture with rHMGB1-stimulated BMDCs developed a Th17 phenotype with increased expression of IL-17A. Third, our findings demonstrate that addition of anti-IL23 into cocultures abrogated the Thl7 response that was induced by rHMGB1-stimulated DCs. Taken together, these observations suggest that generation of Th17 in this BMDC$\mathrm{T}$ cell coculture system is dependent upon the production of the Th17-polarizing cytokine IL-23 by BMDCs in response to rHMGB1.

In addition, we evaluated the effect of anti-HMGB1 IgG on HMGB1 expression, airway inflammation, and AHR in the murine model of neutrophilic asthma. The results showed that administration of anti-HMGB1 IgG before sensitization resulted in decreased HMGB1 expression, low levels of neutrophilic inflammation in lung tissue, little infiltration of the BALF by neutrophils, and decreased AHR. As some studies have shown that Th17 cells mediate neutrophilic airway inflammation and airway hyperresponsiveness in mice [31]; therefore, we postulated that induction of Th17 polarization by HMGB1 might represent a critical initiating event that results in neutrophilic airway inflammation and AHR. In our study, administration of anti-HMGB1 IgG resulted in decreased levels of Th17-related cytokines (IL-23 and IL$17 \mathrm{~A})$ in the BALF and little infiltration of the lung tissue by Th17 cells, suggesting that anti-HMGB1 treatment has an inhibitory effect on the Th17 response. To further explore the mechanism underlying the Th17 skewing effects of HMGB1, we focused on pulmonary DCs, which play a major role in the pathogenesis of asthma and preferentially express RAGE [32], which is a receptor for HMGB1. Because IL-23, which is a Thl7-inducing factor, is usually produced by DCs and macrophages in lung and are sufficient to induce de novo IL17 production from naïve $\mathrm{CD} 4^{+} \mathrm{T}$ cells $[10,33]$, we assessed the number of IL-23-producing CD11c ${ }^{+}$APCs in lung cells. Consistent with the marked reduced level of IL-23 in the BALF, administration of anti-HMGB1 inhibited the number of IL-23-producing $\mathrm{CD}_{11 c^{+}}$APCs in the lung, suggesting that modulation of DC function, including IL-23 production, would be a major mechanism of Th17 suppression by antiHMGB1 IgG.

Last, our study demonstrated that HMGBl's involvement in Th17 polarization in the airways of mice is mediated, at least in part, by airway DCs. Because RAGE is expressed on DCs, monocytes, smooth muscle cells, and endothelial progenitor cells [34], HMGB1 may have direct activation effects on effector cells other than DCs during allergic inflammation. To prove that HMGB1 promotes Th17 polarization predominantly by regulating DCs, we investigated the effects of adoptive transfer of rHMGB1-treated, OVA-pulsed DCs (rHMGB1/OVA-DCs) on airway inflammation in naive mice. 
(A)

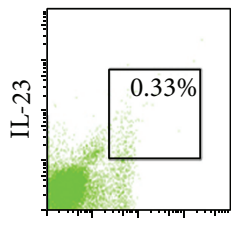

CD11c
(B)

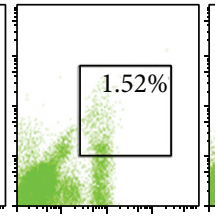

CD11c
(C)

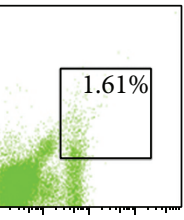

CD11c
(D)

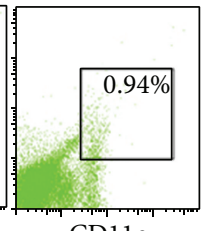

CD11c

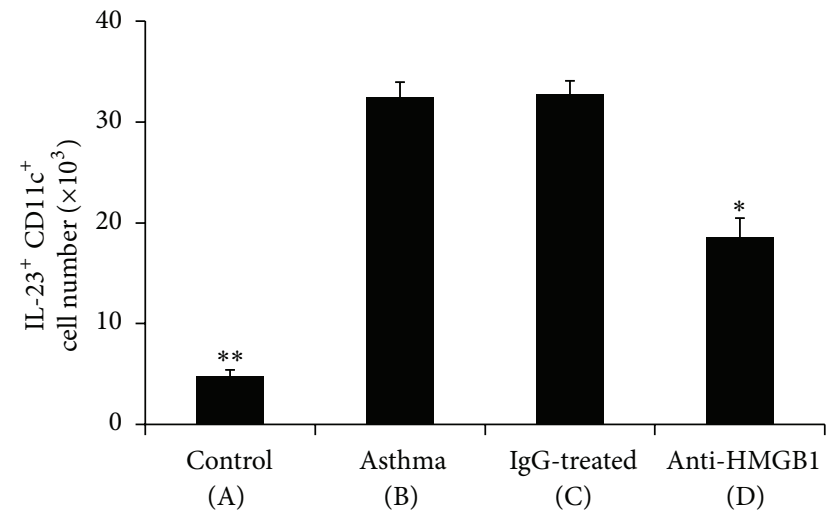

(b)

(a)

FIGURE 7: Intranasal administration of anti-HMGB1 decreased the IL-23 production by lung CD11c ${ }^{+}$APCs. (a) Administration of anti-HMGB1 downregulated the percentage of IL- $23^{+}$CD11c ${ }^{+}$APCs in low-density lung cells. Representative results of the percentage of IL-23 ${ }^{+} \mathrm{CD}_{11 c^{+}}$ APCs in lung from mice in the control (A), asthma (B), control IgG-treated (C), and anti-HMGB1-treated groups (D) were analyzed using FACS. (b) Histogram showing the number of IL-23 ${ }^{+}$CD1lc ${ }^{+}$APCs in mice from the different groups. The values represent the means \pm standard deviations $(n=5) .{ }^{*} P<0.05$ and ${ }^{* *} P<0.01$ compared with the asthma group.

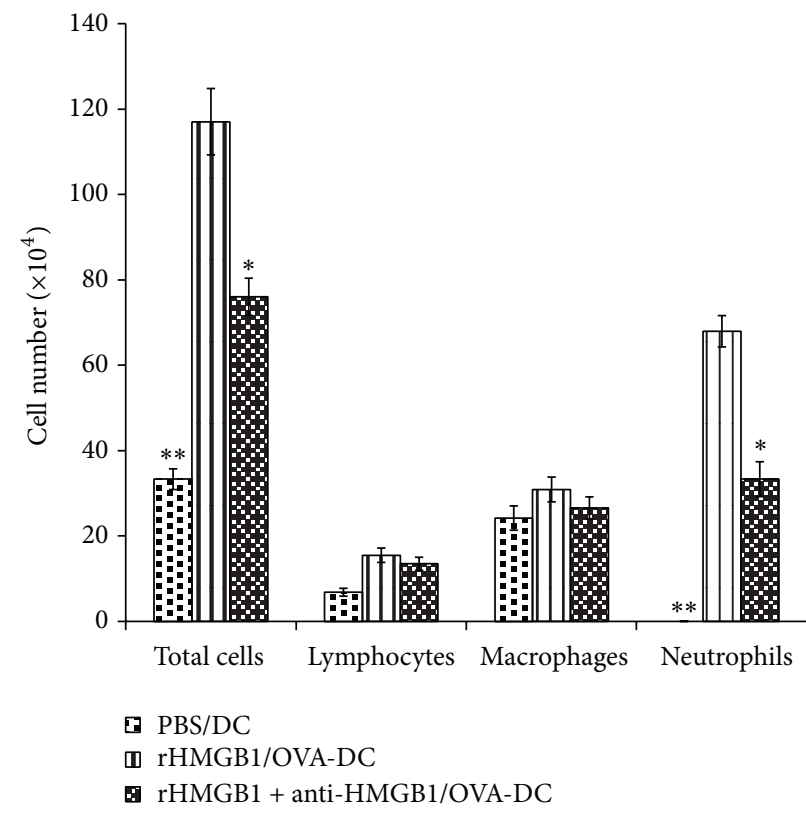

(a)

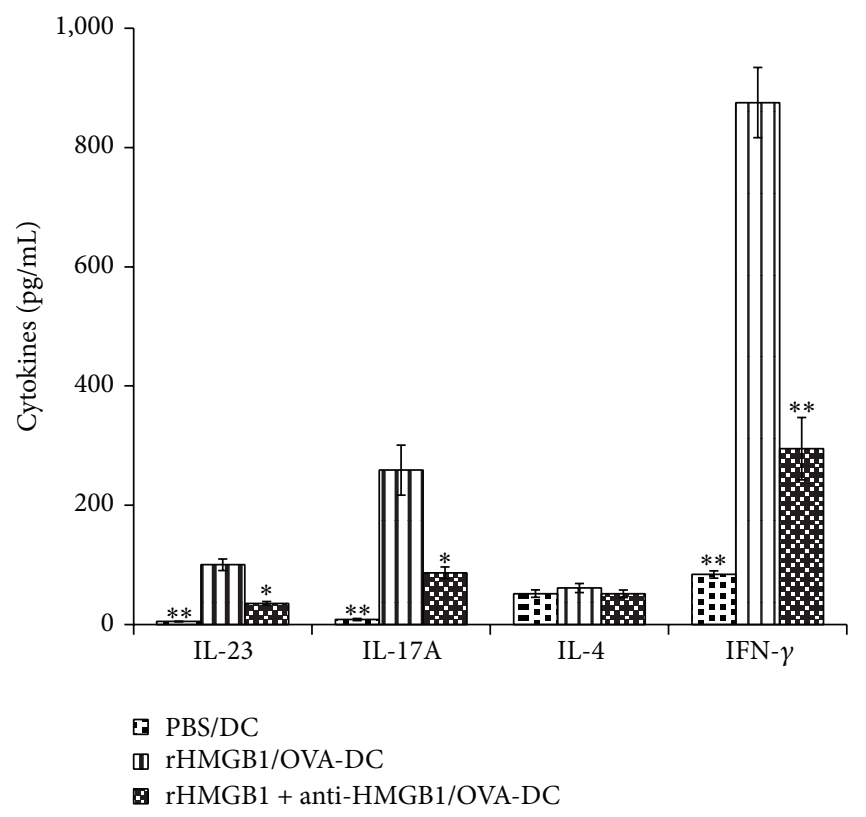

(b)

FIGURE 8: Anti-HMGB1 impaired the ability of HMGB1-stimulated BMDCs to prime Th17 responses in vivo. Mice received an intratracheal (i.t.) injection of $2 \times 10^{6}$ PBS-treated, nonpulsed DCs (PBS/DCs), rHMGB1-treated, OVA-pulsed DCs (rHMGB1/OVA-DCs), or rHMGB1 plus anti-HMGB1-treated, OVA-pulsed DCs (rHMGB1 + anti-HMGB1/OVA-DCs). The mice were then exposed to OVA aerosols on days 10-12. (a) The total and differential cells counts and (b) cytokine profiles in the BALF were assessed $24 \mathrm{~h}$ after the final OVA exposure. The values represent the means \pm standard deviations $(n=5) .{ }^{*} P<0.05$ and ${ }^{* *} P<0.01$ compared with the asthma group.

As expected, adoptive transfer of rHMGB1/OVA-DCs significantly induced neutrophil airway infiltration and a strong Th17 skewing response that was characterized by the release of Th17-related cytokines (IL-23 and IL-17A) in the BALF. However, the adoptive transfer of rHMGB1 plus anti-HMGB1
IgG-treated OVA-DCs (rHMGB1 + anti-HMGB1/OVA-DCs) significantly reduced neutrophil infiltration and Th17-related cytokine secretion in the BALF. These results strongly support the important role of HMGB1 signaling in DC-primed allergic disease and demonstrate that a novel role of HMGB1 
is as a regulator of neutrophilic inflammation and the Th17mediated immune response in airways.

\section{Conclusion}

In the present work, we showed that HMGB1 can contribute to the differentiation of activated $\mathrm{T}$ cells into Th17 cells by inducing BMDCs to produce IL-23 in vitro and that it acts as an immune regulator of the Th17-mediated response in vivo. Anti-HMGB1 IgG suppressed neutrophilic inflammation, AHR, and the Th17-mediated immune response in the lung, at least partly by inhibiting the production of IL23 by lung APCs. Therefore, antagonists of HMGB1 might be promising therapeutic molecules that could treat severe asthma characterized by dominant Th17 phenotypes.

\section{Conflict of Interests}

The authors declare that there is no conflict of interests regarding the publication of this paper.

\section{Acknowledgment}

This work was supported by grants from the National Natural Science Foundation of China (no. 81270079).

\section{References}

[1] J. Deckers, M. F. Branco, and H. Hammad, "Innate immune cells in asthma," Trends in Immunology, vol. 34, no. 11, pp. 540-547, 2013.

[2] D. C. Newcomb and R. S. Peebles Jr., "Th17-mediated inflammation in asthma," Current Opinion in Immunology, vol. 25, no. 6, pp. 755-760, 2013.

[3] D. M. Durrant and D. W. Metzger, "Emerging roles of T helper subsets in the pathogenesis of asthma," Immunological Investigations, vol. 39, no. 4-5, pp. 526-549, 2010.

[4] A. Mishra, X. Yao, and S. J. Levine, "From bedside to bench to clinic trials: identifying new treatments for severe asthma," Disease Models \& Mechanisms, vol. 6, no. 4, pp. 877-888, 2013.

[5] Z. Jaffar, M. E. Ferrini, T. A. Girtsman, and K. Roberts, "Antigenspecific Treg regulate Th17-mediated lung neutrophilic inflammation, B-cell recruitment and polymeric IgA and IgM levels in the airways," European Journal of Immunology, vol. 39, no. 12, pp. 3307-3314, 2009.

[6] S. L. Traves and L. E. Donnelly, "Th17 cells in airway diseases," Current Molecular Medicine, vol. 8, no. 5, pp. 416-426, 2008.

[7] G. Marland, A. B. Bakker, G. J. Adema, and C. G. Figdor, "Dendritic cells in immune response induction," Stem Cells, vol. 14 , no. 5, pp. 501-507, 1996.

[8] E. J. Pearce, C. M. Kane, and J. Sun, "Regulation of dendritic cell function by pathogen-derived molecules plays a key role in dictating the outcome of the adaptive immune response," Chemical Immunology and Allergy, vol. 90, pp. 82-90, 2006.

[9] M. Lagranderie, M. Abolhassani, J. A. Vanoirbeek et al., "Mycobacterium bovis bacillus Calmette-Guérin killed by extended freeze-drying targets plasmacytoid dendritic cells to regulate lung inflammation," Journal of Immunology, vol. 184, no. 2, pp. 1062-1070, 2010.
[10] I. P. Lewkowich, S. Lajoie, J. R. Clark, N. S. Herman, A. A. Sproles, and M. Wills-Karp, "Allergen uptake, activation, and IL-23 production by pulmonary myeloid DCs drives airway hyperresponsiveness in asthma-susceptible mice," PLOS ONE, vol. 3, no. 12, Article ID e3879, 2008.

[11] J. R. Klune, R. Dhupar, J. Cardinal, T. R. Billiar, and A. Tsung, "HMGB1: endogenous danger signaling," Molecular Medicine, vol. 14, no. 7-8, pp. 476-484, 2008.

[12] L. Duan, C. Y. Wang, J. Chen et al., "High-mobility group box 1 promotes early acute allograft rejection by enhancing IL-6-dependent Th17 alloreactive response," Laboratory Investigation, vol. 91, no. 1, pp. 43-53, 2011.

[13] Z. Su, C. Sun, C. Zhou et al., "HMGB1 blockade attenuates experimental autoimmune myocarditis and suppresses Thl7cell expansion," European Journal of Immunology, vol. 41, no. 12, pp. 3586-3595, 2011.

[14] Z. He, S. S. Shotorbani, Z. Jiao et al., "HMGB1 promotes the differentiation of Th17 via up-regulating TLR2 and IL-23 of CD14+ monocytes from patients with rheumatoid arthritis," Scandinavian Journal of Immunology, vol. 76, no. 5, pp. 483-490, 2012.

[15] F. Zhang, Y. Shi, Y. Song et al., "The association of HMGB1 expression and neutrophil account or IL-17 level in the lung of asthmatic mouse," Geriatrics \& Health Care, vol. 18, no. 3, pp. 144-146, 2012.

[16] Y. K. Kim, S. Y. Oh, S. G. Jeon et al., "Airway exposure levels of lipopolysaccharide determine type 1 versus type 2 experimental asthma," Journal of Immunology, vol. 178, no. 8, pp. 5375-5382, 2007.

[17] F. Zhang, G. Huang, B. Hu, Y. Song, and Y. Shi, "A soluble thymic stromal lymphopoietin (TSLP) antagonist, TSLPRimmunoglobulin, reduces the severity of allergic disease by regulating pulmonary dendritic cells," Clinical and Experimental Immunology, vol. 164, no. 2, pp. 256-264, 2011.

[18] Y. S. Kim, S. W. Hong, J. P. Choi et al., "Vascular endothelial growth factor is a key mediator in the development of $\mathrm{T}$ cell priming and its polarization to type 1 and type $17 \mathrm{~T}$ helper cells in the airways," Journal of Immunology, vol. 183, no. 8, pp. 5113-5120, 2009.

[19] F. Zhang, G. Huang, B. Hu, Y. Song, and Y. Shi, "Induction of immune tolerance in asthmatic mice by vaccination with DNA encoding an allergen-cytotoxic T lymphocyte-associated antigen 4 combination," Clinical and Vaccine Immunology, vol. 18, no. 5, pp. 807-814, 2011.

[20] M. Ebina, H. Taniguchi, T. Miyasho et al., "Gradual increase of high mobility group protein b1 in the lungs after the onset of acute exacerbation of idiopathic pulmonary fibrosis," Pulmonary Medicine, vol. 2011, Article ID 916486, 9 pages, 2011.

[21] H. Bai, J. Cheng, X. Gao et al., "IL-17/Th17 promotes type $1 \mathrm{~T}$ cell immunity against pulmonary intracellular bacterial infection through modulating dendritic cell function," Journal of Immunology, vol. 183, no. 9, pp. 5886-5895, 2009.

[22] B. J. Masten, G. K. Olson, D. F. Kusewitt, and M. F. Lipscomb, "Flt3 ligand preferentially increases the number of functionally active myeloid dendritic cells in the lungs of mice," Journal of Immunology, vol. 172, no. 7, pp. 4077-4083, 2004.

[23] X. Lin, H. Yang, T. Sakuragi et al., "Alpha-chemokine receptor blockade reduces high mobility group box 1 protein-induced lung inflammation and injury and improves survival in sepsis," The American Journal of Physiology: Lung Cellular and Molecular Physiology, vol. 289, no. 4, pp. L583-L590, 2005.

[24] L. Lyakh, G. Trinchieri, L. Provezza, G. Carra, and F. Gerosa, "Regulation of interleukin-12/interleukin-23 production and 
the T-helper 17 response in humans," Immunological Reviews, vol. 226, no. 1, pp. 112-131, 2008.

[25] H. Yang and K. J. Tracey, "Targeting HMGB1 in inflammation," Biochimica et Biophysica Acta, vol. 1799, no. 1-2, pp. 149-156, 2010.

[26] V. V. Orlova, E. Y. Choi, C. Xie et al., "A novel pathway of HMGB1-mediated inflammatory cell recruitment that requires Mac-1-integrin," The EMBO Journal, vol. 26, no. 4, pp. 1129-1139, 2007.

[27] R. Bassi, P. Giussani, V. Anelli et al., "HMGB1 as an autocrine stimulus in human T98G glioblastoma cells: role in cell growth and migration," Journal of Neuro-Oncology, vol. 87, no. 1, pp. 23-33, 2008.

[28] P. Rovere-Querini, A. Capobianco, P. Scaffidi et al., "HMGB1 is an endogenous immune adjuvant released by necrotic cells," EMBO Reports, vol. 5, no. 8, pp. 825-830, 2004.

[29] I. E. Dumitriu, P. Baruah, B. Valentinis et al., "Release of high mobility group box 1 by dendritic cells controls T cell activation via the receptor for advanced glycation end products," Journal of Immunology, vol. 174, no. 12, pp. 7506-7515, 2005.

[30] A. A. Manfredi, A. Capobianco, A. Esposito et al., "Maturing dendritic cells depend on RAGE for in vivo homing to lymph nodes," Journal of Immunology, vol. 180, no. 4, pp. 2270-2275, 2008.

[31] H. Wakashin, K. Hirose, I. Iwamoto, and H. Nakajima, "Role of IL-23-Th17 cell axis in allergic airway inflammation," International Archives of Allergy and Immunology, vol. 149, supplement 1, pp. 108-112, 2009.

[32] D. Yang, Q. Chen, H. Yang, K. J. Tracey, M. Bustin, and J. J. Oppenheim, "High mobility group box-1 protein induces the migration and activation of human dendritic cells and acts as an alarmin," Journal of Leukocyte Biology, vol. 81, no. 1, pp. 59-66, 2007.

[33] J. P. Allam, Y. Duan, F. Heinemann et al., "IL-23-producing CD68+ macrophage-like cells predominate within an IL-17polarized infiltrate in chronic periodontitis lesions," Journal of Clinical Periodontology, vol. 38, no. 10, pp. 879-886, 2011.

[34] D. Musumeci, G. N. Roviello, and D. Montesarchio, "An overview on HMGB1 inhibitors as potential therapeutic agents in HMGB1-related pathologies," Pharmacology \& Therapeutics, vol. 141, no. 3, pp. 347-357, 2014. 


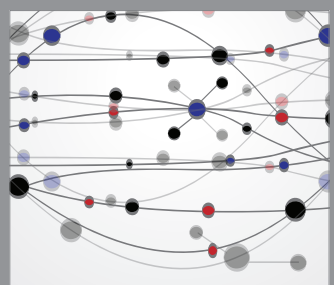

The Scientific World Journal
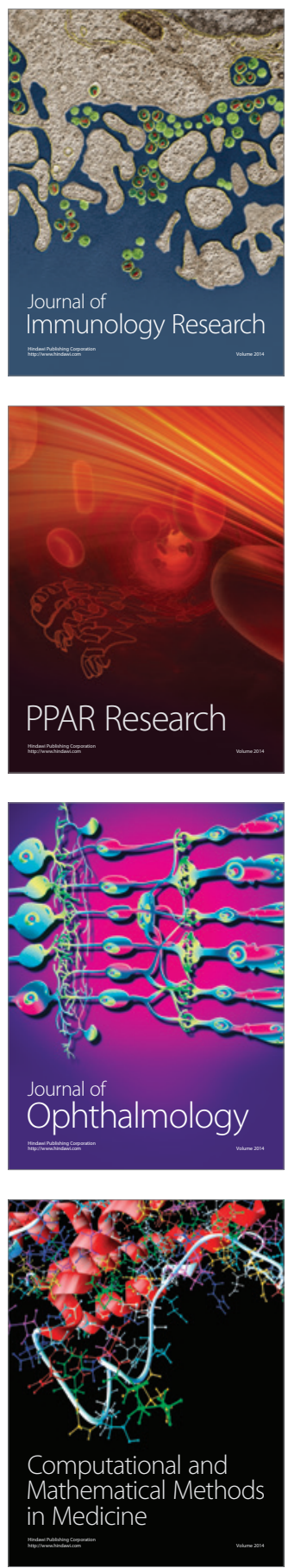

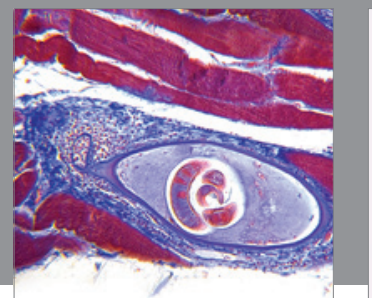

Gastroenterology

Research and Practice
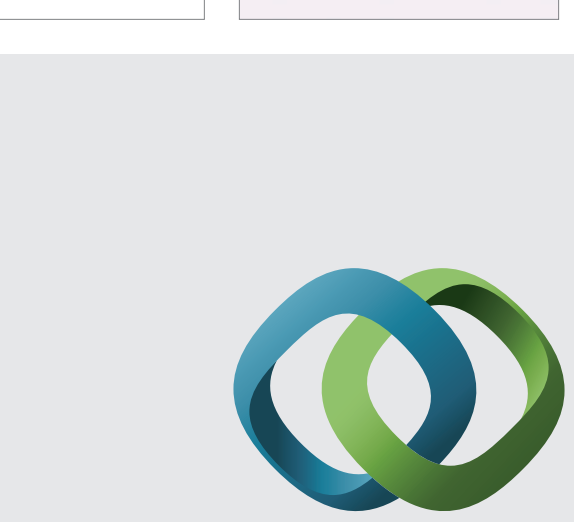

\section{Hindawi}

Submit your manuscripts at

http://www.hindawi.com
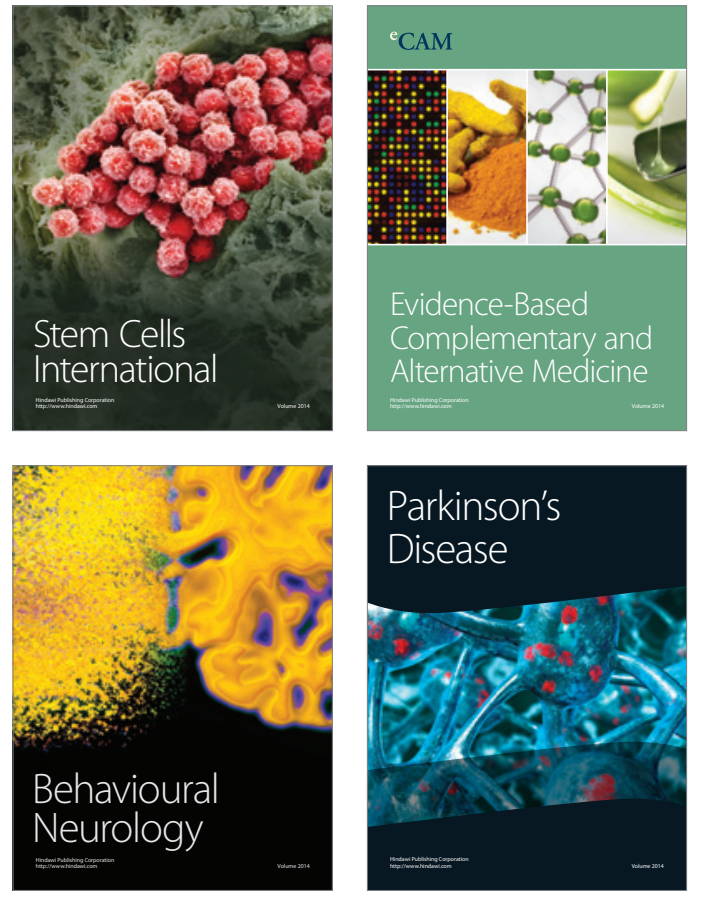
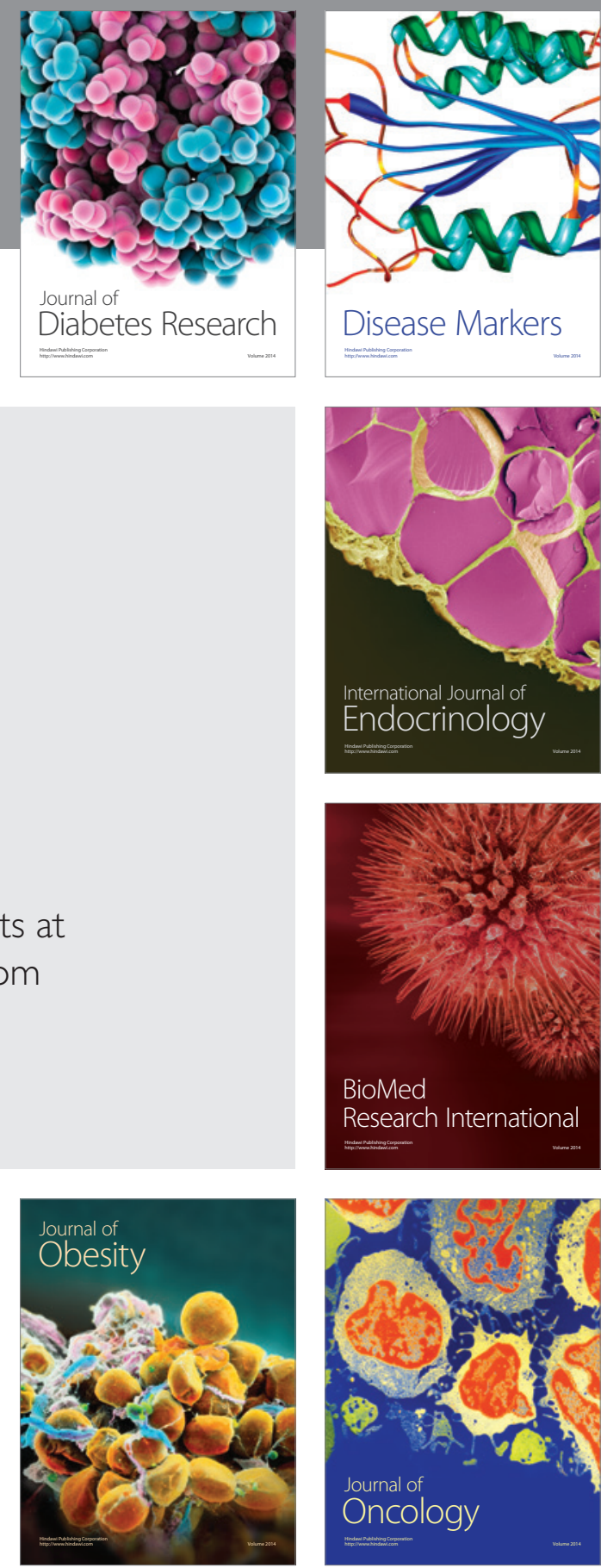

Disease Markers
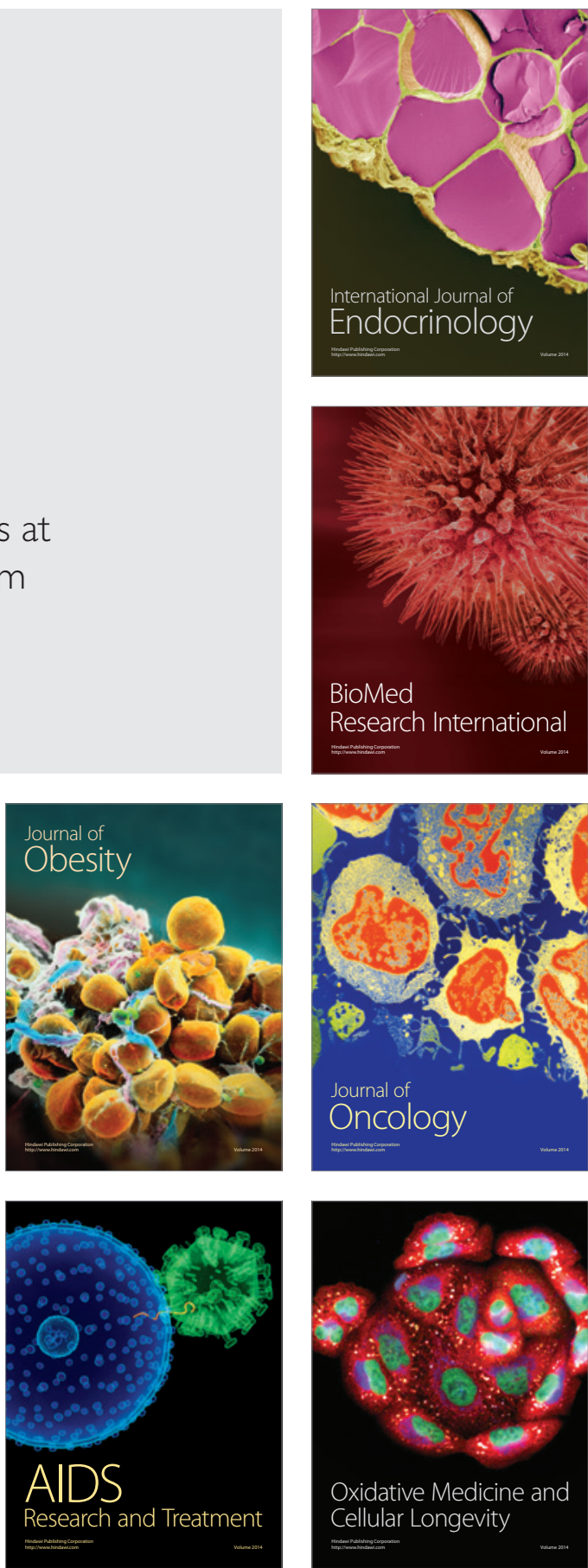